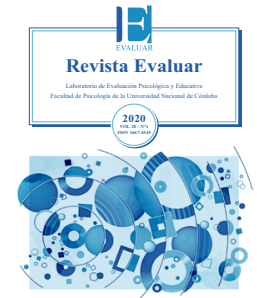

\title{
Desarrollo de instrumentos para medir actitudes hacia la comunidad trans: Análisis preliminares
}

\section{Development of Instruments to Measure Attitudes towards the Trans Community: Preliminary Analysis}

\author{
Caleb Esteban * 1, Juan Aníbal González-Rivera ${ }^{2}$, Margarita Francia-Martínez ${ }^{3}$, Zahira Lespier ${ }^{4}$ \\ 1- Ponce Health Sciences University, Ponce, Puerto Rico. \\ 2- Ponce Health Sciences University, San Juan University Center, Puerto Rico. \\ 3 - Universidad Carlos Albizu, San Juan, Puerto Rico. \\ 4 - Administración de Servicios de Salud Mental y Contra la Adicción, San Juan, Puerto Rico. \\ Recibido: 23/10/2019 Revisado: 15/12/2019 Aceptado: 17/12/2019 \\ Introducción \\ Método \\ Resultados \\ Discusión \\ Conclusión \\ Referencias
}

\section{Resumen}

El propósito de este estudio fue desarrollar y validar preliminarmente dos instrumentos para medir actitudes de psicoterapeutas hacia la comunidad trans. El primer instrumento mide las actitudes de psicoterapeutas hacia la comunidad transgénero y el segundo hacia la comunidad transexual. La muestra contó con 131 participantes reclutados según su disponibilidad. Los resultados confirmaron preliminarmente que ambas escalas poseen una estructura unidimensional. El índice de consistencia interna alfa de Cronbach para la primera escala fue de .75 y de .85 para la segunda. No se encontraron diferencias significativas en las actitudes hacia la comunidad transgénero y transexual entre estudiantes y profesionales de la psicología. Concluimos con que la salud de las personas trans es un derecho humano y universal, por tanto, es responsabilidad de todo psicoterapeuta educarse sobre el tema y es deuda de las instituciones educativas el integrarlo en sus currículos.

Palabras clave: transgénero, transexual, psicoterapia, actitudes, validación de escala

\begin{abstract}
The purpose of this study was to develop and preliminarily validate two instruments to measure attitudes of psychotherapists towards the trans community. The former instrument measures the attitudes of psychotherapists towards the transgender community and the latter, towards the transsexual community. The sample consisted of 131 participants recruited according to their availability. The results preliminarily confirmed that both scales have a one-dimensional structure. Cronbach's alpha internal consistency index for the first scale was .75 and .85 for the second. There were no significant differences in attitudes towards the transgender and transsexual community between students and psychology professionals. We conclude that the health of trans people is a human and universal right, therefore, it is the responsibility of all psychotherapists to educate themselves about the issue and it is a debt of the educational institutions to integrate it into their curriculum.
\end{abstract}

Key words: transgender, transsexual, psychotherapy, attitudes, scale validation

Nota de autor: Expresamos que no hubo conflictos de intereses al redactar el manuscrito. Este artículo es parte del Subcomité de Investigación del Comité de la Diversidad de Sexo, Género y Orientación Sexual de la Asociación de Psicología de Puerto Rico.

* Correspondencia a: Caleb Esteban, Ph.D., Clinical Psychology Program, School of Behavioral and Brain Sciences, Ponce Health Sciences University, PO BOX 7004, Ponce, Puerto Rico 00732-7004. Email: cesteban@psm.edu

Cómo citar este artículo: Esteban, C., González-Rivera, J. A., Francia-Martínez, M., \& Lespier, Z. (2020). Desarrollo de instrumentos para medir actitudes hacia la comunidad trans: Análisis preliminares. Revista Evaluar, 20(1), 67-82. Recuperado de https://revistas.unc.edu.ar/index.php/revaluar 


\section{Introducción}

Las actitudes son respuestas naturales del ser humano (Stangor, 2012). Estas provienen primeramente de una creencia que brinda valores positivos o negativos a un fenómeno social. Por consiguiente, las actitudes son un reflejo de estas creencias que incluye un componente emocional hacia algo o alguien (Lilienfeld, Lynn, Namy, \& Woolf, 2011). Las actitudes juegan un papel vital en la manera en que interactuamos con nuestro entorno. Las mismas han sido objeto de estudio del campo de la psicología social; disciplina que estudia, entre otras cosas, cómo lo cognitivo, lo conductual y lo afectivo interactúan y producen lo que conocemos como experiencia humana.

Con el paso del tiempo, los seres humanos desarrollamos un conjunto de conocimientos que contienen información acerca del yo, de las otras personas, de los grupos sociales, y de los modos de interactuar. Dentro de ese conjunto de conocimientos, se encuentran los esquemas y las actitudes. Los esquemas se refieren mayormente a información acerca de una persona o grupo, mientras que las actitudes se refieren mayormente a una representación que dice si nos gusta o no una persona o grupo social. Cada persona crea unos esquemas que culminan en unas actitudes, esto nos permite pasar juicio rápido sobre si una cosa nos gusta o no, si nos asusta o no, si debemos evadirla o acercarnos, etc. Entonces, las personas pueden interpretar un mismo evento de maneras muy diferentes. Esto hace que la experiencia humana sea interesante, pero también puede acarrear conflictos entre las personas. Entonces, tanto los esquemas como las actitudes influyen en cómo procesamos a nivel cognoscitivo la información social y en cómo nos comportamos (Stangor, 2012).

Según Stangor (2012), el afecto también desempeña un papel importante en la experiencia social humana. Dentro del campo de la psicología social, el afecto hace referencia a los sentimientos que las personas experimentamos en nuestra vida cotidiana. A medida que transcurre el día, podemos experimentar sentimientos de bienestar, de tristeza, de agradecimiento, etc. Si mantenemos nuestros sentimientos regulados adecuadamente, pueden ejercer una función adaptativa, y alertarnos cuando estamos en una situación de potencial peligro. Cuando nos sentimos felices, podemos sentirnos inclinados a interactuar con otros, pero cuando nos sentimos molestos podemos atacar o alejarnos de los demás. De hecho, cuando estamos de buen humor estamos más dispuestos a ayudar a otros y nuestra creatividad aumenta.

Dentro de este marco teórico, las actitudes poseen tres componentes: el afectivo, el cognitivo y el conductual. Estos componentes entran en relación con el entorno sociocultural en el que se desenvuelven las personas y están íntimamente ligados a la manifestación de actitudes que exhibimos hacia los demás, incluyendo a los grupos marginados. Hay algunas actitudes cuyo componente afectivo puede ser mayor, mientras que en otras se puede percibir una prominencia de los aspectos cognitivos o de los comportamentales. Las actitudes también pueden variar su intensidad, aspecto que, en ocasiones, ayuda a predecir nuestras conductas. De ahí que se utilice también este principio para planificar estrategias de mercadeo y publicidad. Si se logra aumentar el sentimiento de bienestar hacia algo, es más probable que eso influya en nuestro comportamiento hacia ese objeto o persona. Si trasladamos estos principios al campo de la psicología clínica, las actitudes positivas hacia una persona o grupo podrían conllevar que nos acerquemos de manera positiva hacia los mismos (Stangor, 2012). 


\section{La comunidad Trans}

La diversidad de las identidades de género es un tema muy discutido en la actualidad (Rodríguez-Madera, Ramos-Pibernus, Padilla, \& Varas-Díaz, 2015). Como fenómeno de estudio, se ha encontrado que existen actitudes negativas hacia personas no-cisgénero o personas que no se identifican con el género asociado al sexo que se les asignó al nacer (Asociación de Psicología de Puerto Rico [APPR], 2014; Ramos-Pibernus, Rodríguez-Madera, Padilla, Varas-Díaz, \& Vargas-Molina, 2016).

Bajo la sombrilla de lo que conocemos como trans-término con que nombramos a las categorías diversas entre los constructos tradicionales de los binomios sociales del sexo y/o el género- tenemos diversas categorías o identidades, tales como: la transgénero, la transexual, la androginia, el género no-binario, el transformismo, el travestismo y, en ocasiones, lo intersexual (APPR, 2014). Muchas de estas categorías han sido establecidas desde centros de investigación con fines analíticos, didácticos y/o categóricos, mientras que otras surgen desde la misma comunidad. Dentro de este grupo de investigación, las comunidades transgénero y transexuales de Puerto Rico han sido el mayor enfoque de estudio y se han encontrado actitudes negativas predominantes en la sociedad (Ramos-Pibernus, 2016; Rodríguez-Madera, \& Toro-Alfonso, 2002, 2003). Sin embargo, cabe destacar que son pocas las investigaciones que se han hecho en esta comunidad y especialmente en el caso de los hombres trans (Ramos-Pibernus et al., 2016; Ramos-Pibernus, 2016).

La comunidad transgénero y la transexual tienen en común la transformación de los conceptos binomiales de nuestra sociedad. Las personas transgénero son aquellas cuya identidad de género, conducta o expresión de género difiere del género relacionado al sexo que les fue asignado en el nacimiento. Las personas transexuales son aquellas que nacieron con una anatomía sexual con la que no se identifican (total o parcialmente), por lo que deciden transformar su corporalidad para experimentar un sentido de congruencia entre la percepción de su género y sus características sexuales primarias y secundarias (APPR, 2014).

Las actitudes negativas se pueden explicar a partir de la tendencia transnegativista de la sociedad en que vivimos (LGBT Advisory Committee, 2011). Esto implica que muchas personas sienten o pueden sentir miedo, odio o demostrar actitudes de prejuicio hacia las personas trans. El desconocimiento, la confusión (Rodríguez-Madera et al., 2015) y la invisibilización de la comunidad (McCann \& Sharek, 2014; Ramos-Pibernus, 2016; Rodríguez-Madera, 2012) son también factores que se añaden al panorama desfavorable. Estas actitudes negativas se manifiestan constantemente en discriminación, aislamiento, hostigamiento o diversos tipos de violencia padecidos por los integrantes de estas comunidades (LGBT Advisory Committee, 2011). A esta visión social se añade el hecho de que muchos profesionales de la psicología y otras disciplinas interpretan la diversidad de la identidad de género como una patología clínica (Jorge, 2016).

\section{Antecedentes de medición}

En Argentina, Páez, Hevia, Pesci y Rabbia (2015) construyeron una escala que mide actitudes negativas hacia personas trans. La primera parte de este estudio contó con 203 participantes, en su mayoría estudiantes universitarios, y en ella se llevaron a cabo la construcción del instrumento y análisis exploratorios. La segunda parte contó con 408 participantes de la población general seleccionados mediante muestreo por cuotas. En esta 
se hicieron análisis factoriales y de validación externa del instrumento. El resultado final fue una escala de 9 reactivos, con una consistencia interna aceptable $(\alpha=.88)$. Además, se encontró relación entre actitudes negativas y religiosidad, mayor edad, mayor asistencia al culto, menor educación, mayor dominancia social y mayor autoritarismo. En los Estados Unidos de América, Norton y Herek (2013) llevaron a cabo un estudio de muestra probabilística con personas heterosexuales. En este estudio se utilizaron varias escalas para medir las actitudes hacia las personas transgénero y otros grupos minoritarios. Para medir las actitudes hacia la comunidad trans, utilizaron una medición de termómetro. En este tipo de medición, se solicita a los participantes que indiquen su disposición hacia varias comunidades a partir de una escala de 0 a 100, donde 0 corresponde a las actitudes más negativas y 100 a las más positivas. Estos autores encontraron que las actitudes de los hombres heterosexuales eran más negativas hacia las personas trans. Además, estas actitudes negativas se asociaban con niveles altos de autoritarismo, conservacionismo político y religiosidad. En Canadá, Ali, Fleisher y Erickson (2015) midieron las actitudes de psiquiatras y residentes en psiquiatría. Mediante un estudio en línea, obtuvieron una muestra de 142 participantes. Utilizaron la Escala de Ideología de Género y Transfobia (GTS) que mide sentimientos, pensamientos y conductas antitransgénero. Este cuestionario había sido publicado anteriormente (Hill \& Willoughby, 2005) y traducido al español (Carrera-Fernández, Lameiras-Fernández, Rodríguez-Castro, \& Vallejo-Medina, 2014). Consta de 32 reactivos que se responden mediante una escala Likert, en la cual puntuaciones más altas significan mayor intolerancia hacia la no conformidad del género. Ali et al. (2016) encontraron puntuaciones más bajas que en un estudio con estudiantes universitarios publicado previamente. Además, encontra- ron relaciones asociadas a la identidad de género, ideología política y religiosidad.

En Australia, Riggs y Sion (2016) exploraron las actitudes de personas cisgénero, profesionales de salud mental. En tres diferentes estudios, los autores examinaron estudiantes de bachillerato $\mathrm{y}$, en los dos estudios restantes, profesionales de salud mental. Las muestras fueron de 173, 122 y 304 participantes, respectivamente. Para los primeros dos estudios se utilizó la Escala de Ideología de Género y Transfobia (GTS) adaptada al contexto australiano (GTS-RA, por sus siglas en inglés). En el primer estudio con estudiantes universitarios $(\alpha=.94)$, los hombres resultaron con puntuaciones más altas en los tres factores (sentimientos, pensamientos y conductas). En el segundo estudio en que participaron psicólogos, trabajadores sociales y consejeros $(\alpha=.87)$, los hombres también puntuaron más alto. Por último, en el tercer estudio que contó con psicólogos, trabajadores sociales, consejeros, psiquiatras y enfermeras en salud mental, se utilizó una versión modificada del GTS-RA, llamada Escala de Actitudes de Consejeros hacia Personas Transgénero. Esta escala utiliza una escala Likert de seis puntos y cuenta con 20 reactivos $(\alpha=.92)$. Este estudio igualmente encontró que las actitudes de los hombres eran más negativas.

En Estados Unidos, Walzer (2015) investigó las actitudes y los conocimientos sobre el estudiantado transgénero. Participaron 121 psicólogos escolares que realizaban su práctica en un ambiente escolar en los estados de New Jersey, Delaware, Connecticut y Maryland. Para este estudio se creó una escala de 20 reactivos llamada Knowlege and Attitudes Toward Transgender Student Survey $(\alpha=.78)$. En los resultados no se encontró una diferencia significativa por el sexo del psicólogo escolar ni tampoco una diferencia por la edad. Sin embargo, se encontró una diferencia significativa en los resultados de aquellos participantes que es- 
taban familiarizados con la posición del National Association of School Psychologists en comparación con los que no.

\section{Propósito del estudio}

Grant et al. (2011) realizaron un estudio en EEUU con 6450 personas identificadas como trans y género no-binario, y encontraron que el $86 \%$ de su muestra informó haber experimentado abuso físico y sexual, y discriminación en el empleo, acoso escolar, hostigamiento, pérdida de relaciones, pérdida de hogares y negación a ofrecerle servicios médicos. Además, la comunidad trans suele sufrir de doble discriminación, tanto por personas heterosexuales como por parte de la comunidad de lesbianas, gays y bisexuales. Esta discriminación no solo proviene de la comunidad en general, sino que también se han registrado experiencias negativas de la comunidad trans en los espacios de psicoterapia. Por lo tanto, cuando acuden a buscar ayuda, en muchas ocasiones continúan recibiendo experiencias de estigma, discriminación y transfobia (Bockting, Miner, Swinburne-Romine, Hamilton, \& Coleman, 2013). Por ende, se vuelve necesario medir las actitudes hacia la comunidad trans, con el propósito de evaluar qué se debe trabajar y educar.

Actualmente, según nuestra revisión de la literatura, no existen escalas para medir las actitudes de los psicoterapeutas hacia la comunidad trans. Tomando esto en consideración, el presente trabajo tuvo como propósito desarrollar y validar preliminarmente dos instrumentos para medir actitudes de psicoterapeutas hacia la comunidad trans. Se decidió crear dos escalas diferentes, ya que las actitudes respecto a la transgeneridad y a la transexualidad pueden variar porque refieren a constructos diferentes. La transgeneridad alude a los constructos sociales del género y la transexualidad, a los biocorporales del sexo. El primer instrumento mide las actitudes de psicoterapeutas hacia la comunidad transgénero; es decir, aquellas personas cuya identidad, expresión de género o conductas (total o parcialmente) no se ajustan a las normas y expectativas típicamente asociadas con el sexo que se les ha asignado al nacer (APPR, 2014). Por su parte, el segundo instrumento evalúa las actitudes de psicoterapeutas hacia la comunidad transexual; es decir, personas que nacieron con una anatomía sexual con la que no se sienten identificadas (total o parcialmente), por lo que deciden o desean transformar su corporalidad para experimentar un sentido de congruencia entre la percepción de su género y sus características sexuales primarias y secundarias (APPR, 2014). Con este fin, se examinó la validez de constructo y la confiabilidad de los dos instrumentos. Y, como objetivo secundario, se propuso evaluar si existían diferencias en las actitudes hacia la comunidad trans entre estudiantes y profesionales de la psicología.

\section{Método}

\section{Diseño de investigación}

Esta investigación tiene un diseño de estudio instrumental (Ato, López, \& Benavente, 2013; Montero \& León, 2007). Se desarrollaron dos instrumentos para medir actitudes de psicoterapeutas hacia la comunidad trans y se obtuvieron sus propiedades psicométricas preliminares.

\section{Participantes}

Se trabajó con una muestra no probabilística constituida por profesionales de la psicología $(n=92)$ y estudiantes graduados de psicología ( $n$ = 39), que decidieron confidencial y voluntaria- 
mente participar del estudio. La edad promedio de la muestra fue de 37.27 años (DE = 12.84). En la Tabla 1 se presentan los datos sociodemográficos de los participantes. Los criterios de inclusión fueron: (a) ser mayor de 21 años de edad y (b) tener experiencia o práctica supervisada en intervenciones de psicoterapia o evaluaciones psicológicas.

Tabla 1

Datos sociodemográficos de la muestra.

\begin{tabular}{lll}
\hline Variable & f & $\%$ \\
\hline
\end{tabular}

$\begin{array}{lcc}\text { Sexo } & & \\ \text { Hombre } & 42 & 32.1 \\ \text { Mujer } & 89 & 67.9 \\ & & \\ \text { Género } & & \\ \text { Masculino } & 42 & 32.1 \\ \text { Femenino } & 88 & 67.2 \\ \text { Otro } & 1 & 0.8\end{array}$

\section{Orientación sexual}

Heterosexual

$104 \quad 79.4$

Homosexual

$19 \quad 14.5$

Bisexual

Pansexual

Valores perdidos

$5 \quad 3.8$

10.8

$2 \quad 1.5$

\section{Estado Civil}

Solteros

$48 \quad 36.6$

Casados

$34 \quad 26.0$

Convivencia

$18 \quad 13.7$

Divorciados

$16 \quad 12.2$

Noviazgo

$13 \quad 9.9$

Viudos

$2 \quad 1.5$

\section{Religión}

Católica

Protestante

$62 \quad 47.3$

Evangélica

Otra
Ninguna
Valores perdidos

$37 \quad 28.2$

\section{Valores perdidos}

Título académico en Psicología

Doctorado en Psicología (PsyD)

Doctorado en Filosofía (PhD)

Maestría en Ciencias (MS)

Maestría en Artes (MA)

Estudiante graduado

Valores Perdidos

$2 \quad 1.5$

Nota. $n=131$.

\section{Instrumentos}

Cuestionario de datos generales. Para identificar las características sociodemográficas de la muestra se desarrolló un cuestionario de datos generales que recogía información importante con respecto a la edad, sexo, género, estado civil, orientación sexual y religión.

\section{Escala de Actitudes de Psicoterapeutas hacia la Comunidad Transgénero. Este instrumento fue} diseñado por los investigadores con el propósito de desarrollar una justa medida que examine las actitudes de estudiantes y profesionales de la psicología hacia la comunidad transgénero. Con este fin, se desarrollaron 43 ítems que recogen varias posibles actitudes hacia la comunidad transgénero, tales como: empatía, cercanía, incomodidad, conocimiento, apertura, prejuicios, aceptación, entre otros. Los ítems fueron sometidos a la opinión de 10 jueces conocedores y estudiosos de la comunidad LGBT en Puerto Rico, con el objetivo de identificar si los ítems del instrumento son pertinentes al constructo que se desea medir (método de Lawshe). Para rechazar o retener los ítems se utilizó la razón de validez de contenido (CVR) y para interpretar los resultados, los valores crí- 
ticos recalculados por Wilson, Pan y Schumsky (2012). De acuerdo con estos autores, el valor mínimo requerido para 10 jueces, según la tabla de Schipper, es de .62 para la aceptación de un ítem como esencial. Solo 25 ítems obtuvieron valores aceptables, por lo que permanecieron en el instrumento preliminar. La escala de respuesta del instrumento es tipo Likert de cuatro puntos: 1 (Totalmente de acuerdo), 2 (Parcialmente de acuerdo), 3 (Parcialmente en desacuerdo) y 4 (Totalmente en desacuerdo). Contiene ítems que son de corrección inversa. A mayor puntuación, mayores actitudes negativas (prejuicios) hacia personas transgénero.

\section{Escala de Actitudes de Psicoterapeutas hacia la} Comunidad Transexual. Este instrumento fue diseñado por los investigadores con el propósito de desarrollar una justa medida que examine las actitudes de estudiantes y profesionales de la psicología hacia la comunidad transexual. También se desarrollaron 43 ítems que recogen los mismos aspectos que el instrumento anterior, pero dirigidos a personas transexuales. Los ítems fueron sometidos a la opinión de 10 jueces conocedores y estudiosos de la comunidad LGBT en Puerto Rico (método de Lawshe). Para rechazar o retener los ítems se utilizaron la razón de validez de contenido (CVR) y los valores críticos recalculados por Wilson et al. (2012). En esta ocasión, 30 ítems obtuvieron valores aceptables, por lo que permanecieron en el instrumento preliminar. La escala de respuesta del instrumento es tipo Likert de cuatro puntos: 1 (Totalmente de acuerdo), 2 (Parcialmente de acuerdo), 3 (Parcialmente en desacuerdo) y 4 (Totalmente en desacuerdo). Igualmente, contiene ítems que son de corrección inversa. A mayor puntuación, mayores actitudes negativas (prejuicios) hacia personas transexuales.

\section{Procedimientos}

La investigación fue aprobada por el Institutional Review Board (IRB) de la Universidad Carlos Albizu de Puerto Rico (Fall-14-09). Luego se procedió a recopilar la información de manera presencial y en línea, a través del portal surveymonkey.com. Se utilizó una hoja de consentimiento informado, en la que no era necesario identificarse con nombre, para notificar a los participantes del propósito del estudio, su naturaleza voluntaria, los posibles riesgos y beneficios, así como de su derecho a retirarse del estudio en cualquier momento. Además, se informó sobre la duración de su participación y su derecho a conocer los resultados del estudio. Los acercamientos presenciales se realizaron mediante visitas a centros de trabajo de profesionales de la psicología, así como visitas a centros universitarios donde cursan estudiantes graduados de psicología en Puerto Rico.

\section{Análisis de datos}

Una vez recopilados los datos, los analizamos utilizando el sistema para análisis estadísticos IBM SPSS v. 24 (IBM Corp., 2016). En este programa se realizaron análisis descriptivos de la muestra, análisis de ítems para conocer su índice de discriminación, análisis de factores y análisis de confiabilidad para calcular el coeficiente alfa de Cronbach y división en mitades de Spearman-Brown. Además, se realizó un análisis de correlación $r$ de Pearson entre las dos escalas. Valores inferiores a .35 se consideran correlaciones débiles o bajas, valores entre .36 y .67 se consideran correlaciones moderadas, valores entre .68 y .89 se consideran correlaciones altas y, por último, valores de .90 en adelante se consideran correlaciones muy altas (Taylor, 1990). El tamaño 
del efecto de la correlación se calculó mediante el coeficiente de determinación $r^{2}$. Por último, se efectuó un análisis de comparación de medias prueba $t$ con el propósito de evaluar si existen diferencias estadísticamente significativas entre el estudiantado y los profesionales de la psicología en términos de las actitudes hacia la comunidad trans. El tamaño del efecto de la prueba $t$ se calculó con la $d$ de Cohen.

Para el análisis factorial exploratorio, se utilizó el método de extracción de mínimos cuadrados no ponderados (unweighted least squares) con rotación oblicua para identificar las variables latentes que subyacen en los ítems. Se utilizó este procedimiento de ajuste por dos razones principales: (1) el método de extracción de mínimos cuadrados no ponderados es el más recomendado actualmente para trabajar con muestras pequeñas, especialmente si el número de factores a retener es pequeño (Jung, 2013) y evita la aparición de casos Heywood (saturaciones mayores que la unidad y varianzas de error negativas), más frecuentes con otros métodos de estimación (Lloret-Segura, Ferreres-Traver, Hernández-Baeza, \& Tomás-Marco, 2014); y (2) la rotación oblicua es más precisa y provee más información que la rotación octogonal (Schmitt, 2011).

Para determinar el número de factores se utilizó la cantidad de varianza explicada mediante el número de factores extraídos. Hatcher (1994) sugiere que los factores a considerar sean aquellos que al menos expliquen un 5\% de la varianza. Comúnmente, se considera que un análisis factorial es aceptable si la suma de las varianzas de los factores que se retienen es igual o mayor al 50\% (Ferré, 1995; Streiner, 1994). No obstante, en ciertos análisis factoriales exploratorios, pueden ser admisibles soluciones factoriales que expliquen al menos el $30 \%$ de la varianza total (Macía-Sepúlveda, 2010). Como criterios de aceptación, consideramos aquellos ítems con una carga factorial mayor a .30 en un solo factor (Kline, 2000). Para conocer la capacidad de discriminación de los ítems de los factores, se calculó el índice de correlación ítem total (rbis). La literatura identifica como índices adecuados de discriminación valores mayores que .30 y menores que 70 (Field, 2013). Para conocer el grado de confiabilidad de la escala, se calcularon el coeficiente alfa de Cronbach, alfa de Cronbach estandarizado y el coeficiente de división en mitades de Spearman-Brown. Field (2013) indica que índices mayores a .70 son aceptables.

\section{Resultados \\ Validez de construcción lógica}

Actitudes hacia la comunidad transgénero. Se realizó un primer análisis factorial exploratorio para evaluar la adecuación de los datos e identificar los factores que explican el 5\% o más de la varianza para ser retenidos en la escala. La prueba de Kaiser-Meyer-Olkin apoyó la adecuación de los datos de muestreo para el análisis: KMO $=.719$. La prueba de esfericidad de Bartlett fue significativa $\left(\chi^{2}(300)=728.939, p<.001\right)$, indicando que las correlaciones entre los ítems fueron significativamente diferentes de cero y es un indicador adicional de la adecuación para el análisis factorial. Considerando los criterios de selección de ítems, se evidenció la retención de un solo factor, ya que los factores 2 en adelante explicaban menos del 5\% de la varianza o no contaban con suficientes ítems. Al realizar el análisis restringido a un factor, de los 25 ítems se eliminaron 15 porque no cumplieron con los criterios de selección. Este procedimiento redujo la escala a 10 ítems que explican el 33\% de la varianza de los datos originales. En la Tabla 2 se puede apreciar las cargas factoriales de esta versión de la escala. 
Actitudes hacia la comunidad transexual. Se realizó un análisis factorial exploratorio para evaluar la adecuación de los datos e identificar los factores que explican el 5\% o más de la varianza para ser retenidos en la escala. La prueba de Kaiser-Meyer-Olkin apoyó la adecuación de los datos de muestreo para el análisis: $\mathrm{KMO}=.734$. La prueba de esfericidad de Bartlett fue significativa $\left(\chi^{2}(435)=1454.164, p<.001\right)$. Considerando los criterios de selección de ítems, se evidenció la retención de un solo factor. Al realizar el análisis restringido a un factor, de los 30 ítems se eliminaron 20 que no cumplieron con los criterios de selección. Este procedimiento redujo la escala a 10 ítems que explican el 44\% de la varianza de los datos originales (véase Tabla 2).

\section{Análisis de distribución de items}

Con los datos obtenidos se llevaron a cabo análisis de ítems para determinar los índices de discriminación de cada instrumento. La Escala de Actitudes de Psicoterapeutas hacia la Comunidad Transgénero mostró índices de discriminación que fluctuaban entre .36 y .55 . Por su parte, la Escala de Actitudes de Psicoterapeutas hacia la Comunidad Transexual evidenció índices de discriminación que fluctuaban entre .44 y .66. Todos los valores están dentro de los puntos de corte recomendados por la literatura. En la Tabla 2 se presentan los índices de discriminación por ítem de cada escala.

\section{Análisis de confiabilidad y correlación}

Los ítems de cada escala fueron sometidos a un análisis de consistencia interna para determinar el índice de confiabilidad de los instrumentos. Para esto se utilizaron dos métodos: el coefi- ciente alfa de Cronbach y la división en mitades de Spearman-Brown. La Escala de Actitudes de Psicoterapeutas hacia la Comunidad Transgénero mostró un coeficiente alfa de $.75 \mathrm{y}$ de .76 para la prueba Spearman-Brown. Por su parte, la Escala de Actitudes de Psicoterapeutas hacia la Comunidad Transexual evidenció un coeficiente alfa de .85 y de .81 para la prueba Spearman-Brown. El análisis de correlación $r$ de Pearson evidenció una asociación alta, positiva y estadísticamente significativa entre ambas escalas $(r=.70, p<.001$, $\left.r^{2}=.49\right)$

Diferencias entre profesionales y estudiantes de psicología

Se realizó un análisis de comparación de medias mediante prueba $t$ con el propósito de evaluar si existen diferencias estadísticamente significativas entre las medias de actitudes hacia la comunidad trans entre profesionales de la psicología y estudiantes graduados de psicología (véase Tabla 4). Los análisis reflejaron que no existen diferencias estadísticamente significativas en las actitudes hacia la comunidad transgénero y transexual entre profesionales de la psicología y estudiantes graduados de psicología.

\section{Discusión}

Lamentablemente, existe suficiente evidencia como para afirmar categóricamente que la comunidad trans es blanco de un continuo rechazo, prejuicio y discrimen (Francia-Martínez, Esteban, \& Lespier, 2017). El desconocimiento, la confusión y la invisibilización de la comunidad puede provocar que los profesionales de la psicología asuman actitudes negativas hacia personas transexuales y transgénero. No obstante, ni Puer- 
Tabla 2

Índice de discriminación, varianza explicada de los ítems y cargas factoriales.

\begin{tabular}{lccclccc}
\hline $\begin{array}{l}\text { Escala de Actitudes de Psicoterapeutas hacia la } \\
\text { Comunidad Transgénero }\end{array}$ & \multicolumn{5}{l}{$\begin{array}{l}\text { Escala de Actitudes de Psicoterapeutas hacia la } \\
\text { Comunidad Transexual }\end{array}$} \\
\hline Ítem & rbis & $\mathrm{R}^{2}$ & Factor 1 & Ítem & rbis & $\mathrm{R}^{2}$ & Factor 1 \\
\hline 2 & .55 & .38 & .64 & 30 & .55 & .37 & .60 \\
4 & .36 & .24 & .44 & 38 & .44 & .25 & .48 \\
12 & .38 & .27 & .44 & 41 & .59 & .46 & .64 \\
15 & .42 & .28 & .51 & 44 & .62 & .47 & .67 \\
17 & .42 & .23 & .45 & 47 & .47 & .26 & .51 \\
19 & .40 & .38 & .59 & 50 & .59 & .45 & .64 \\
20 & .49 & .26 & .50 & 52 & .50 & .39 & .56 \\
23 & .44 & .28 & .41 & 53 & .62 & .48 & .68 \\
24 & .36 & .57 & .46 & 54 & .61 & .87 & .66 \\
25 & .42 & .57 & 55 & .66 & .88 & .71 \\
\hline
\end{tabular}

Nota. rbis $=$ índice de discriminación del ítem; $R^{2}=$ varianza explicada de los ítems; Factor $1=$ cargas del análisis de factores exploratorio. $(n=131)$.

Tabla 3

Medias, desviaciones estándar, alfas y correlaciones entre las escalas.

\begin{tabular}{lccccccc}
\hline Factor & M & DE & $\boldsymbol{\alpha}$ & aest & Spearman-Brown & $\mathbf{1}\left(\boldsymbol{r}^{2}\right)$ & $\mathbf{2}(\boldsymbol{r})$ \\
\hline 1.Transgénero & 13.25 & 3.96 & .75 & .77 & .76 & - & .70 \\
2.Transexual & 14.94 & 5.38 & .85 & .86 & .81 & .49 & - \\
\hline
\end{tabular}

Nota. $\mathrm{M}=$ media; $\mathrm{DE}=$ desviación estándar; $\alpha=$ alfa de Cronbach; $\alpha$ est $=$ alfa de Cronbach estandarizado. La correlación sobre la diagonal representa la correlación $r$ de Pearson, mientras que la correlación bajo la diagonal representa el coeficiente de determinación $r^{2}$. Todas las correlaciones fueron significativas a $p<.001$. $(\mathrm{n}=131)$.

\section{Tabla 4}

Resultados del análisis de comparación de medias prueba $t$ y tamaño del efecto.

\begin{tabular}{lccccccc}
\hline & $\mathbf{N}$ & $\mathbf{M}$ & $\mathbf{D E}$ & $\boldsymbol{t}$ & $\boldsymbol{g l}$ & $\boldsymbol{p}$ & $\boldsymbol{d}$ \\
\hline Actitudes hacia Transgénero & & & & & & & \\
Profesionales & 87 & 13.22 & 4.14 & .107 & 122 & .92 & .02 \\
Estudiantes & 37 & 13.14 & 3.45 & & & & \\
$\begin{array}{l}\text { Actitudes hacia Transexual } \\
\text { Profesionales }\end{array}$ & & & & & & & .10 \\
Estudiantes & 89 & 15.10 & 5.68 & .500 & 125 & .62 & .10 \\
\hline
\end{tabular}

Nota. $\mathrm{N}=$ participantes; $\mathrm{M}=$ medias; $g l=$ grados de libertad; $\mathrm{DE}=$ desviación estándar; $t=$ valor de la prueba $\mathrm{t}$; $p=$ significancia; $d=$ tamaño del efecto $d$ de Cohen. $(\mathrm{N}=131)$. 
to Rico ni el Caribe contaban con un instrumento diseñado para medir las actitudes de los psicoterapeutas hacia la comunidad trans. Es por ello que el presente estudio tuvo como propósito desarrollar, validar y examinar las propiedades psicométricas de dos instrumentos en una muestra de profesionales y estudiantes graduados de psicología: (a) Escala de Actitudes de Psicoterapeutas hacia la Comunidad Transgénero; y (b) Escala de Actitudes de Psicoterapeutas hacia la Comunidad Transexual. Los resultados obtenidos confirman preliminarmente que ambos instrumentos poseen propiedades psicométricas adecuadas para medir actitudes favorables o desfavorables hacia las personas trans.

En términos teóricos, los análisis factoriales exploratorios revelaron la existencia de un solo factor latente en ambos instrumentos. Esto evidencia cómo el prejuicio hacia la comunidad trans, tanto hacia transexuales como hacia transgénero, es consistente en sus expresiones, formas y manifestaciones. Sorprendentemente, un 20\% de los participantes evidenció puntuaciones correspondientes a un nivel alto de prejuicio hacia la comunidad trans. Esto sugiere que educarse formalmente en psicología puede colaborar en el mantenimiento de un discurso académico de inclusión, pero no asegura que en el ámbito personal $-y$, en ocasiones, en el laboral- los profesionales de la psicología mantengan conductas coherentes con la inclusión y la aceptación.

Por su parte, los análisis comparativos revelaron que no existen diferencias estadísticamente significativas en las actitudes hacia la comunidad trans entre estudiantes y profesionales de la psicología. Esto puede implicar, entre otras cosas, que el prejuicio hacia las personas trans no se debe a un asunto generacional ni de experiencia profesional. Este hallazgo nos da a entender que los años de experiencia en psicoterapia por sí solos no mejoran las actitudes de prejuicio hacia la co- munidad trans en los y las psicoterapeutas. Además, la falta de capacitación en el tema en el país aporta a la falta de introspección, conocimiento y competencias clínicas de los y las psicoterapeutas para trabajar con las identidades de género (Esteban-Reyes, Ortíz-Mendoza, Rivera-Morales, Purcell-Baerga, \& Ruiz-Mojica, 2016).

En cuanto a la confiabilidad de las escalas, se obtuvieron índices superiores al mínimo recomendado por la literatura científica (Field, 2013; Kline, 2000). Esto sugiere preliminarmente que tanto la Escala de Actitudes de Psicoterapeutas hacia la Comunidad Transgénero como la Escala de Actitudes de Psicoterapeutas hacia la Comunidad Transexual son instrumentos estables, reproducibles y consistentes en la medida de prejuicio hacia las personas trans. Asimismo, las correlaciones de cada ítem con la puntuación total manifiestan una consistencia interna notable. Esto sugiere que los ítems de ambos instrumentos discriminan adecuadamente y son capaces de diferenciar personas con distintos niveles de prejuicio hacia la comunidad trans.

En cuanto a las implicaciones prácticas, ambas escalas son de las primeras que se construyen en español en Latinoamérica y las primeras en Centro América y el Caribe que miden actitudes hacia la población trans. Además, son las únicas escalas en español que miden actitudes hacia esta población en el proceso de psicoterapia. Instrumentos como estos son sumamente importantes para el desarrollo de la psicología latinoamericana y el avance hacia una psicoterapia auténticamente inclusiva. Las escalas no solo miden los niveles de actitudes de los terapeutas hacia la comunidad trans, sino que también demuestran la necesidad de formación en el tema y las repercusiones en psicoterapia sobre la transignorancia (Francia-Martínez, Esteban, \& Lespier, 2017). Es responsabilidad de todo terapeuta formarse en el tema y deuda de las instituciones educativas, 
integrarlo en sus currículos académicos (Coleman et al., 2012). La comunidad trans no puede continuar siendo un sector marginado, juzgado ni discriminado, especialmente tratándose de profesionales de salud mental como los profesionales de la psicología.

En términos de corrección, las puntuaciones de las escalas se calculan mediante la sumatoria de los 10 ítems para obtener un índice general. En los apéndices A y B se encuentran las escalas finales con sus respectivas normas de corrección, ya que varios ítems se corrigen de forma inversa. Al finalizar la sumatoria, se clasifican las puntuaciones de acuerdo a los cuartiles. Las puntuaciones de la Escala de Actitudes de Psicoterapeutas hacia la Comunidad Transgénero se categorizan de la siguiente manera: ningún prejuicio (puntuación de 10), prejuicio bajo (de 11 a 15) y prejuicio alto ( de 16 a 40). Las puntuaciones de la Escala de Actitudes de Psicoterapeutas hacia la Comunidad Transexual se categorizan de la siguiente forma: ningún prejuicio (puntuación de 10), prejuicio bajo (de 11 a 17) y prejuicio alto (de 16 a 40).

\section{Limitaciones y recomendaciones}

El estudio tuvo la limitación metodológica de ser realizado con una muestra por disponibilidad relativamente pequeña. No obstante, se tomó en consideración el tamaño de la muestra y el nivel de significancia al momento de tomar decisiones sobre los ítems, así como un método de extracción adecuado a dicha limitación (Jung, 2013). Otra limitación fue que no se pudo establecer la confiabilidad del instrumento a través del tiempo, solo se pudo hacer a través de sus elementos. Se recomienda para futuros estudios llevar a cabo una muestra probabilística para evitar cualquier sesgo en los participantes. También, se recomiendan análisis estadísticos adicionales para mejorar los diversos tipos de confianza y validez de la escala. Además, se recomienda ampliar la muestra a otros países y otras disciplinas que ofrecen psicoterapia, terapia o consejería. Es necesario crear escalas para medir actitudes en otros profesionales de la salud mental y de la salud en general.

\section{Conclusión}

Tanto la Escala de Actitudes de Psicoterapeutas hacia la Comunidad Transgénero como la Escala de Actitudes de Psicoterapeutas hacia la Comunidad Transexual mostraron preliminarmente indicadores adecuados de validez y confiabilidad, lo que sugiere que presentan el potencial para avanzar en la investigación con miras a comprender con mayor amplitud el fenómeno del prejuicio hacia la comunidad trans entre los profesionales de la psicología. Se espera que dichos instrumentos sean beneficiosos en el ámbito de la investigación y faciliten nuevos conocimientos sobre este fenómeno en Puerto Rico y América Latina.

\section{Referencias}

Ali, N., Fleisher, W., \& Erickson, J. (2016). Psychiatrists' and psychiatry residents' attitudes toward transgender people. Academic Psychiatry, 40(2), 268-273. doi: 10.1007/s40596-015-0308-y

Asociación de Psicología de Puerto Rico. (2014). Estándares para el trabajo e intervención en comunidades lesbianas, gay, bisexuales e identidades trans (LGBT). San Juan, Puerto Rico: Autor.

Ato, M., López, J. J., \& Benavente, A. (2013). Un sistema de clasificación de los diseños de investigación en 
psicología. Anales de Psicología, 29(3), 1038-1059. doi: 10.6018/analesps.29.3.178511

Bockting, W. O., Miner, M. H., Swinburne-Romine, R. E., Hamilton, A., \& Coleman, E. (2013). Stigma, mental health, and resilience in an online sample of the US transgender population. American Journal of Public Health, 103(5), 943-951. doi: 10.2105/ AJPH.2013.301241

Carrera-Fernández, M. V., Lameiras-Fernández, M., Rodríguez-Castro, Y., \& Vallejo-Medina, P. (2014). Spanish adolescents' attitudes toward transpeople: Proposal and validation of a short form of the Genderism and Transphobia Scale. The Journal of Sex Research, 51(6), 654-666. doi: 10.1080/00224499.2013.773577

Coleman, E., Bockting, W., Botzer, M., Cohen-Kettenis, P., DeCuypere, G., Feldman, J., ... Zucker, K. (2012). Standards of care for the health of transsexual, transgender and gender-nonconforming people. International Journal of Transgenderism, 13(4), 165-232. doi: 10.1080/15532739.2011.700873

Esteban-Reyes, C., Ortiz-Mendoza, C. M., Rivera-Morales, N., Purcell-Baerga, P., \& Ruiz-Mojica, G. (2016). La educación del género en peligro de extinción: Preparación de psicoterapeutas clínicos en Puerto Rico. Revista Puertorriqueña de Psicología, 27(1), 80-93. Recuperado de http://www.ojs.repsasppr.net/index. $\mathrm{php} / \mathrm{reps} / \mathrm{index}$

Ferré, L. (1995). Selection of components in principal component analysis: A comparison of methods. Computational Statistics \& Data Analysis, 19(6), 669-682. doi: 10.1016/0167-9473(94)00020-j

Field, A. P. (2013). Discovering statistics using IBM SPSS statistics ( $4^{\mathrm{a}}$ ed.). London, England: SAGE.

Francia-Martínez, M., Esteban, C., \& Lespier, Z. (2017). Actitudes, conocimiento y distancia social de psicoterapeutas con la comunidad transgénero y transexual. Revista Puertorriqueña de Psicología, 28(1), 98-113. Recuperado de http://www.ojs.repsasppr. net/index.php/reps

Grant, J. M., Mottet, L. A., Tanis, J., Harrison, J. L., Herman, J., \& Keisling, M. (2011). Injustice at every turn: A report of the National Transgender Discrimination Survey. Recuperado de https://transequality. org

Hatcher, L. (1994). A step-by-step approach to using the SAS system for factor analysis and structural equation modeling. Cary, NC: SAS Institute.

Hill, D. B., \& Willoughby, B. L. B. (2005). The development and validation of the Genderism and Transphobia Scale. Sex Roles, 53(7-8), 531-544. doi: 10.1007/ s11199-005-7140-X

IBM Corp. (2016). IBM SPSS Statistics for Windows (version 24.0). [software de cómputo] Armonk, NY: Autor.

Jorge, J. C. (2016). El nombrar clínico: La disforia de género. Boletín Diversidad, 7(1), 6-9. Recuperado de https://www.boletindiversidad.org

Jung, S. (2013). Exploratory factor analysis with sma11 sample sizes: A comparison of three approaches. Behavioural Processes, 97(1), 90-95. doi: 10.1016/j. beproc.2012.11.016

Kline, P. (2000). The Handbook of Psychological Testing ( $2^{\mathrm{a}}$ ed.). New York, NY: Routledge.

LGBT Advisory Committee \& San Francisco Human Rights Commission. (2011). Bisexual invisibility: Impacts and recommendations. San Francisco, CA: San Francisco Human Rights Commission. Recuperado de https://sf-hrc.org

Lilienfeld, S. O., Lynn, S. J., Namy, L. L., \& Woolf, N. J. (2011). Psicología: Una introducción. Madrid, España: Pearson Education.

Lloret-Segura, S., Ferreres-Traver, A., Hernández-Baeza, A., \& Tomás-Marco, I. (2014). El análisis factorial exploratorio de los ítems: Una guía práctica, revisada y actualizada. Anales de Psicología, 30(3), 11511169. doi: 10.6018/analesps.30.3.199361

Macía-Sepúlveda, F. (2010). Validez de los tests y el análisis factorial: Nociones generales. Ciencia \& Trabajo, 12(35), 276-280. Recuperado de https://dialnet.unirioja.es/servlet/articulo? codigo $=3218921$

McCann, E., \& Sharek, D. (2014). Survey of lesbian, gay, bisexual and transgender people's experiences of 
mental health services in Ireland. International Journal of Mental Health Nursing, 23(2), 118-127. doi: 10.1111/inm. 12018

Montero, I., \& León, O. G. (2007). A guide for naming research studies in psychology. International Journal of Clinical and Health Psychology, 7(3), 847-862. Recuperado de https://aepc.es/ijchp/busca.php

Norton, A. T., \& Herek, G. M. (2013). Heterosexuals' attitudes toward transgender people: Findings from a national probability sample of U.S. adults. Sex Roles, 68, 738-753. doi: 10.1007/s11199-011-0110-6

Páez, J., Hevia, G., Pesci, F., \& Rabbia, H. H. (2015). Construcción y validación de una escala de actitudes negativas hacia personas trans. Revista de Psicología, 33(1), 151-188. Recuperado de http://revistas.pucp. edu.pe/index.php/psicologia

Ramos-Pibernus, A. G. (2016). Hombres trans en Puerto Rico: Una comunidad escondida. Boletín Diversidad, 7(1), 10-11. Recuperado de http:/www.boletindiversidad.org

Ramos-Pibernus, A. G., Rodríguez-Madera, S. L., Padilla, M., Varas-Díaz, N., \& Vargas-Molina, R. (2016). Intersections and evolution of 'Butch-trans' categories in Puerto Rico: Needs and barriers of an invisible population. Global Public Health, 11(7-8), 966-980. doi: 10.1080/17441692.2016.1180703

Riggs, D. W., \& Sion, R. (2016). Gender differences in cisgender psychologists' and trainees' attitudes toward transgender people. Psychology of Men \& Masculinities, 18(2), 187-190. doi: 10.1037/men0000047

Rodríguez-Madera, S. L. (2012). TRANS-acciones de la carne: Criminalización de mujeres trans puertorriqueñas que ejercen el trabajo sexual. En S. M. Serrano-Rivera (Ed.), Registros Criminológicos Contemporáneos (pp. 107-150). San Juan, Puerto Rico: Situm.

Rodríguez-Madera, S., Ramos-Pibernus, A., Padilla, M., \& Varas-Díaz, N. (2015). Radiografías de las comunidades trans en Puerto Rico: Visibilizando femineidades y masculinidades alternas. En M. Vázquez-Rivera, A. Martínez-Taboas, M. Francia-Martínez \& J.
Toro-Alfonso (Eds.), LGBT 101: Una mirada introductoria al colectivo (pp. 315-342). Hato Rey, Puerto Rico: Publicaciones Puertorriqueñas.

Rodríguez-Madera, S., \& Toro-Alfonso, J. (2002). Ser o no ser: La transgresión del género como objeto de estudio de la psicología. Avances en Psicología Clínica Latinoamericana, 22, 63-78.

Rodríguez-Madera, S., \& Toro-Alfonso, J. (2003). La comunidad de la cual no hablamos: Vulnerabilidad social, conductas de riesgo y VIH/SIDA en la comunidad de transgénero en Puerto Rico. Revista Puertorriqueña de Psicología, 14(1), 7-40. Recuperado de http:// www.ojs.repsasppr.net/index.php/reps/index

Schmitt, T. A. (2011). Current methodological considerations in exploratory and confirmatory factor analysis. Journal of Psychoeducational Assessment, 29(4), 304-321. doi: 10.1177/0734282911406653

Stangor, C. (2012). Social Psychology Principles. Recuperado de https://opentextbc.ca/socialpsychology/\#main

Streiner, D. L. (1994). Figuring out factors: The use and misuse of factor analysis. The Canadian Journal of Psychiatry, 39(3), 135-140. doi: $10.1177 / 070674379403900303$

Taylor, R. (1990). Interpretation of the correlation coefficient: A basic review. Journal of Diagnostic Medical Sonography, 6(1), 35-39. doi: 10.1177/875647939000600106

Walzer, A. (2015). School psychologists'knowledge of and attitudes towards transgender students (Tesis de maestría). Recuperado de https://rdw.rowan.edu/etd

Wilson, F. R., Pan, W., \& Schumsky, D. A. (2012). Recalculation of the critical values for Lawshe's content validity ratio. Measurement and Evaluation in Counseling and Development, 45(3), 197-210. doi: $10.1177 / 0748175612440286$ 


\section{Apéndice A}

Escala de Actitudes de Psicoterapeutas hacia la Comunidad Transgénero

\begin{tabular}{|l|l|l|l|l|}
\hline & $\begin{array}{c}\text { Totalmente } \\
\text { de acuerdo }\end{array}$ & $\begin{array}{c}\text { Parcialmente } \\
\text { de acuerdo }\end{array}$ & $\begin{array}{c}\text { Parcialmente } \\
\text { en desacuerdo }\end{array}$ & $\begin{array}{c}\text { Totalmente en } \\
\text { desacuerdo }\end{array}$ \\
\hline $\begin{array}{l}\text { 1. Si puedo escoger, evito tener que atender a clientes } \\
\text { transgénero. }\end{array}$ & & & & \\
\hline $\begin{array}{l}\text { 2. Se me hace difícil hablar de las relaciones sexuales o de } \\
\text { pareja con un/a cliente transgénero. }\end{array}$ & & & & \\
\hline $\begin{array}{l}\text { 3. Creo inadecuado que una persona transgénero asista a } \\
\text { una actividad donde haya niños/as. }\end{array}$ & & & \\
\hline $\begin{array}{l}\text { 4. Reconozco que la transgresión del género reta mis va- } \\
\text { lores personales. }\end{array}$ & & & \\
\hline $\begin{array}{l}\text { 5. Me sentiría tranquilo/a si mis hijos/as tuvieran un mae- } \\
\text { stro/a transgénero. }\end{array}$ & & & \\
\hline $\begin{array}{l}\text { 6. Entiendo que darles derechos y reconocimiento a las } \\
\text { personas transgénero trastoca los valores de nuestra so- } \\
\text { ciedad. }\end{array}$ & & & & \\
\hline $\begin{array}{l}\text { 7. Considero que las personas transgénero que deciden } \\
\text { salir a la calle vestida/os del otro género, son ejemplo de } \\
\text { superación y valentía. }\end{array}$ & & & & \\
\hline 8. Considero que las personas transgénero son inestables. & & & & \\
\hline 9. Compartiría mi oficina con un/a terapeuta transgénero. & & & & \\
\hline $\begin{array}{l}\text { 10. Estoy de acuerdo que las personas transgéneros, } \\
\text { puedan cambiar todos sus documentos, con su identidad } \\
\text { de género nueva. }\end{array}$ & & & & \\
\hline
\end{tabular}

\section{Método de corrección sumaria}

Puntuación regular: los ítems se puntúan de 1 (Totalmente de acuerdo) a 4 (Totalmente en desacuerdo). Puntuación inversa: los ítems 1, 2, 3, 4, 6 y 8 se puntúan de forma inversa, de 4 (Totalmente de acuerdo) a 1 (Totalmente en desacuerdo). 


\section{Apéndice B}

Escala de Actitudes de Psicoterapeutas hacia la Comunidad Transexual

\begin{tabular}{|c|c|c|c|c|}
\hline & $\begin{array}{l}\text { Totalmente } \\
\text { de acuerdo }\end{array}$ & $\begin{array}{l}\text { Parcialmente } \\
\text { De acuerdo }\end{array}$ & $\begin{array}{l}\text { Parcialmente } \\
\text { en desacuerdo }\end{array}$ & $\begin{array}{l}\text { Totalmente en } \\
\text { desacuerdo }\end{array}$ \\
\hline $\begin{array}{l}\text { 1. Se me hace difícil hablar de las relaciones sex- } \\
\text { uales o de pareja con un/a cliente transexual. }\end{array}$ & $\square$ & $\square$ & $\square$ & $\square$ \\
\hline $\begin{array}{l}\text { 2. Me sentiría tranquilo/a si mis hijos/as tuvieran } \\
\text { un/a maestro/a transexual. }\end{array}$ & $\square$ & $\square$ & $\square$ & $\square$ \\
\hline $\begin{array}{l}\text { 3. Reconozco que la transexualidad reta mis va- } \\
\text { lores personales. }\end{array}$ & $\square$ & $\square$ & $\square$ & $\square$ \\
\hline $\begin{array}{l}\text { 4. Me sentiría tranquilo/a si alguno de mis hijos/as } \\
\text { fuera transexual. }\end{array}$ & $\square$ & $\square$ & $\square$ & $\square$ \\
\hline $\begin{array}{l}\text { 5. Entiendo que darles derechos y reconocimiento } \\
\text { a las personas transexuales trastoca los valores } \\
\text { de nuestra sociedad. }\end{array}$ & $\square$ & $\square$ & $\square$ & $\square$ \\
\hline $\begin{array}{l}\text { 6. Me sentiría tranquilo/a si alguno de mis famili- } \\
\text { ares fuera transexual. }\end{array}$ & $\square$ & $\square$ & $\square$ & $\square$ \\
\hline $\begin{array}{l}\text { 7. Compartiría mi oficina con un/a terapeuta tran- } \\
\text { sexual. }\end{array}$ & $\square$ & $\square$ & $\square$ & $\square$ \\
\hline $\begin{array}{l}\text { 8. Apoyo el que las personas transexuales puedan } \\
\text { cambiar todos sus documentos con su identidad } \\
\text { de género nueva. }\end{array}$ & $\square$ & $\square$ & $\square$ & $\square$ \\
\hline $\begin{array}{l}\text { 9. Para mí una mujer transexual (hombre a mujer) } \\
\text { es una mujer como cualquier otra. }\end{array}$ & $\square$ & $\square$ & $\square$ & $\square$ \\
\hline $\begin{array}{l}\text { 10. Para mí un hombre transexual (mujer a hombre) } \\
\text { es un hombre como cualquier otro. }\end{array}$ & $\square$ & $\square$ & $\square$ & $\square$ \\
\hline
\end{tabular}

\section{Método de corrección sumaria}

Puntuación regular: los ítems se puntúan de 1 (Totalmente de acuerdo) a 4 (Totalmente en desacuerdo). Puntuación inversa: los ítems 1, 3 y 5 se puntúan de forma inversa, de 4 (Totalmente de acuerdo) a 1 (Totalmente en desacuerdo). 